\title{
Epidemiología delcáncer de ovario en el hospital nacional de clínicas. Universidad nacional de córdoba, 2014-2020.
}

\section{Epidemiology of ovary cancer At the clinic national hospital. National university of cordoba. 2014-2020.}

\author{
DOI: $10.46932 /$ sfjdv2n3-030
}

Received in: May 1st, 2021

Accepted in: Jun 30th, 2021

\section{Claudia Guerrero}

Dra en Medicina y Cirugía. Investigadora y profesora de la Facultad de Ciencias Médicas

(UNC. IUCBC. UNVM)

\section{Patricia Pelliccioni}

Dra en Bioquímica. Investigadora y profesora de la Facultad de Ciencias de la Salud (UCC).

\section{Karen Rhys}

Odontóloga. Investigadora y profesora de la Facultad de Ciencias de la Salud

(UCC, UNC y UAP).

\section{Mabel Brunotto}

Dra en Biología.

Profesora de la Facultad de Odontología (UNC).

\section{Julieta Imahorn}

Médica residente del Servicio de Patológica del Hospital Nacional de Clínicas (UNC)

Natalia Saavedra

Estudiante de Medicina. (IUCBC)

\section{María E Ruggieri}

Estudiante de Medicina. (IUCBC)

\section{María Elisa Dionisio Cabalier}

Doctora en Medicina y Cirugía. Profesora y Jefe del Servicio de Patología (UNC)

\section{RESUMEN}

Introducción: El objetivo del presente trabajo fue describir características anatomopatológicas, imagenológicas y clínicas de pacientes diagnosticadas de cáncer de ovario (CO) del Hospital Nacional de Clínicas (HNC) dependiente de la Universidad Nacional de Córdoba (UNC) entre 2014 a 2020. Métodos: Estudio retrospectivo, descriptivo. Basado en la revisión de 26 historias clínicas de pacientes diagnosticadas de tumores sólidos de ovario, las muestras se colorearon con técnicas de hematoxilinaeosina y se utilizaron los inmuno marcadores ck7, ck20, EMA, cd20. De este grupo se detectó y estudió un caso con diagnóstico de $\mathrm{CO}$.

Resultados: En la serie de tumores estudiados la mediana etaria correspondió a 63 años. La ocurrencia de $\mathrm{CO}$ en este estudio fue $3,8 \%$. La imagenología evidenció compromiso poliorgánico y los subtipos 
histológicos establecieron el pronóstico. Conclusión: La interpretación de los resultados debe tener en cuenta: clínica, imagenología, histología e inmunohistoquímica. Dado lo complejo del diagnóstico el consejo genético cobra importancia.

Palabras claves: cáncer de ovario, epidemiología, inmunohistoquímica.

\begin{abstract}
Introduction: The objective of this study was to describe anatomopathological, imaging and clinical characteristics of patients diagnosed with ovarian cancer in the Hospital Nacional de Clínicas (HNC) dependent of the Universidad Nacional de Córdoba (UNC) between 2014 to 2020. Methods: Retrospective, descriptive and epidemiological study. Based

on the review of 26 clinical records of patients diagnosed with solid ovarian tumors, histological techniques were analyzed using hematoxylin-eosin techniques and using the ck7, ck20, EMA, cd20 immunomarkers. A case diagnosed with $\mathrm{CO}$ was detected and studied from this group. Results: In the series of tumors studied, the median age corresponds to 63 years old. The $\mathrm{CO}$ incidence in this study was $3.8 \%$. Imaging showed polyorganic compromise and the histological subtypes established the prognosis. Conclusion: The results interpretation should be into account: clinical, imaging, histology, and immunohistochemistry. Facing complexity of the diagnosis, genetic advice becomes important.
\end{abstract}

Key words: ovarian cancer, epidemiology, immunohistochemistry.

\title{
1 INTRODUCCIÓN
}

En la Argentina, el cáncer de ovario constituye la 6ta causa de mortalidad por cáncer en mujeres, con una tasa bruta de mortalidad cercana a 5 por cada 100000 habitantes. El 50\% de los casos ocurren en mujeres mayores de 65 años. Sólo una muy pequeña mejora en la supervivencia global de CO se ha realizado en los últimos 25 años ${ }^{(1,2)}$. Esta elevada tasa de mortalidad y la falta de progreso en el tratamiento de esta enfermedad se deben, en parte, al frecuente diagnóstico de la enfermedad a nivel avanzado, aproximadamente, 2/3 de las pacientes se encuentran en estadío avanzado en el momento del diagnóstico.

Su mayor incidencia se presenta en mujeres entre 75 y 79 años, ya que se desarrolla principalmente en mujeres postmenopáusicas,

La mayor expectativa de vida en la población ha aumentado el número de casos de esta neoplasia. El cáncer de ovario tiene un curso silente en sus etapas iniciales, lo que hace que la mayoría de los casos sean diagnosticados en estadios avanzados, con lo que la sobrevida promedio a 5 años es de 40 a $50 \%{ }^{(3)}$ correspondiendo el $90 \%$ a carcinoma epitelial de ovario. ${ }^{(4)}$ El cáncer de ovario ocupa el $6^{\circ}$ lugar entre las neoplasias malignas que afectan a las mujeres, y supone el $4 \%$ del total. Su incidencia es mayor en los países industrializados (América del Norte y Europa) ${ }^{(5,6)}$ 
Las neoplasias ováricas se distinguen histológicamente y se dividen en tumores epiteliales, tumores de células germinativas y tumores del cordón sexual-estromal, según el tipo de célula que le dio origen.

Las mujeres con tumores en estadio I (confinados al ovario) tienen una sobrevida a 5 años de más de 70 a $80 \%$ mientras que en los estadios III y IV (diseminación extrapélvica) sólo llega a 5 ó 10\%.A partir de estos datos se sugiere que la detección temprana del cáncer de ovario CO podría mejorar el pronóstico de las pacientes diagnosticadas con esta patología y en este sentido los estudios ecográficos son importantes. ${ }^{(7)}$ Sin embargo, el diagnóstico temprano es complicado por el hecho de que no existe una prueba efectiva de detección temprana ${ }^{(8,9,10)}$.

El objetivo del presente trabajo es describir las características anatomopatológicas, imagenológicas y clínicas de pacientes con diagnóstico de $\mathrm{CO}$, según consta en la historia clínica del Hospital Nacional de Clínicas (UNC) en el período comprendido entre los años 2014 a 2020.

\section{MATERIAL Y MÉTODOS}

El diseño del estudio fue retrospectivo y descriptivo, basado en la revisión de historias clínicas de las pacientes atendidas en el (HNC) de la UNC, desde el 1 de enero de 2014 al 31 de diciembre de 2020 con diagnóstico de tumores sólidos de ovario, según los criterios de la Federación Internacional de Ginecología y Obstetricia (FIGO) ${ }^{(11)}$.

La muestra final quedó constituida por 26 casos que tenían historias clínicas completas a fin de permitir el análisis. A partir de este grupo se identificó una paciente con diagnóstico de CO.

Los estudios de anatomía patológica se realizaron con las técnicas de rutina, procesados con alcoholes ascendentes, incluidos en parafina, cortes con micrótomo de 4 micras y coloreados con hematoxilina-eosina (HE). Se utilizó inmunomarcación con los siguientes reactivos: ck7, ck20, EMA, ki67,cd20.

En relación con los resultados de inmunohistoquímica (IHQ), se evaluó la tinción por 2 patólogos, sin conocimiento del diagnóstico en cada caso, según intensidad y porcentaje de células positivas, llegándose a un consenso en todos los casos. Para la intensidad se consideró: negativo (sin señal), débil (tinción suave), intenso (señal acentuada). Para el porcentaje de células teñidas se consideró señal negativa: $0 \%$ de células positivas, +: hasta 10\%, ++: hasta 50\%, +++: más de 50\% de las células positivas. Para la evaluación final de los resultados, éstos se agruparon en 3 categorías:

- Negativo: tinción negativa, tinción débil +;

- Positivo débil: tinción débil ++, tinción débil +++;

- Positivo intenso: tinción intensa ++, tinción intensa +++ 


\section{ANÁLISIS ESTADÍSTICO DE DATOS}

Los datos cuantitativos se describieron mediantes medidas de posición: media, mediana y percentiles y por su error estándar (medida de dispersión). Mientras que los datos cualitativos fueron descriptos por sus frecuencias absolutas.

\section{COMITÉ DE ÉTICA}

Esta investigación realizada bajo Ley 9694 de la Provincia de Córdoba queda excluida de la aprobación de un comité de ética porque no incluye intervenciones sobre la salud humana y no supone riesgo para los individuos, por ser un estudio retrospectivo.

Conflicto de interés: Ninguno. La responsabilidad del trabajo es sólo de los autores.

Fuentes de financiamiento: No hubo fuente de financiación

\section{RESULTADOS}

En nuestra serie del total de tumores sólidos de ovario estudiados $(n=26)$, sólo uno correspondió a CO. Su tipo histológico fue indiferenciado, esto establece parámetros para su pronóstico ya que se caracterizan por su agresividad biológica.

El gráfico 1 A muestra la ocurrencia de $\mathrm{CO}$ (carcinoma poco diferenciado) en el total de los tumores sólidos analizados y correspondió al 3,8\% de la muestra total. Por otra parte, la relación entre el diagnóstico de tumores sólidos de ovario y la edad de las pacientes se muestran en el gráfico $1 \mathrm{~B}$; en donde se observa que las pacientes presentaron una mediana de edad de 63 años \pm 3años

Gráfico 1. A. Incidencia de CO (carcinoma poco diferenciado) en relación a los tumores sólidos de ovario analizados en el Hospital Nacional de Clínicas. B. Casos de tumores sólidos en relación a la edad. Las líneas representan el percentil 25 (53 años); el percentil 50 (63 años) y el percentil 75 (67 años).

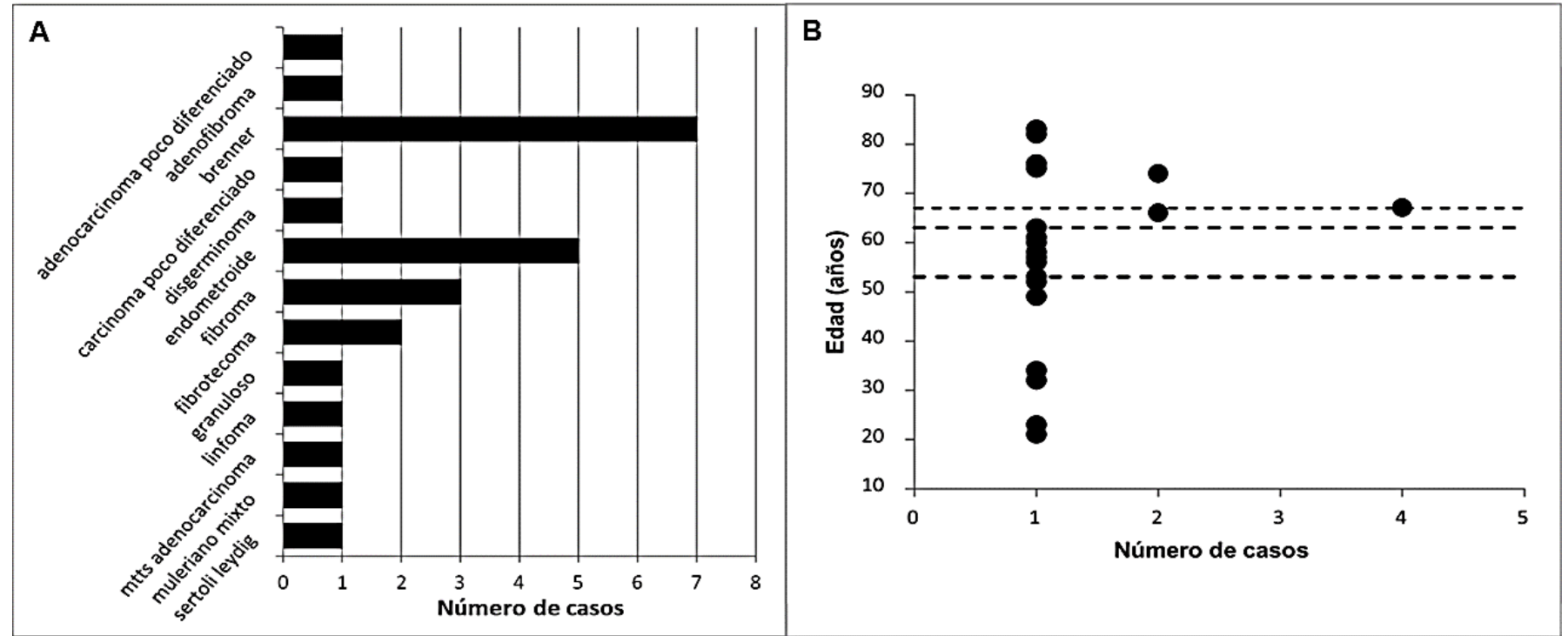


La Tabla 1 resume las características estudiadas, haciendo especial énfasis en los hallazgos de anatomía patológica. El caso analizado correspondió a una recidiva de 2 años de evolución donde los estudios de imágenes mostraron compromiso orgánico que excedía al ovario.

Los aspectos clínicos consignados en la historia del paciente se relacionan al compromiso local.

Tabla 1: Cáncer de Ovario indiferenciado: descripción clínica, anatomopatológica e imagenológica.

\begin{tabular}{|c|c|c|}
\hline $\begin{array}{c}\text { CÁNCER DE OVARIO } \\
n=1\end{array}$ & Tipo & Poco diferenciado \\
\hline \multirow{5}{*}{ CLÍNICA } & Edad & 67 \\
\hline & Motivo de Consulta & Recidiva de CO a los 2 años. \\
\hline & Procedencia & Córdoba \\
\hline & Síntomas & Cambio de hábito intestinal \\
\hline & Antecedentes obstétricos & G:3 \\
\hline \multirow{8}{*}{$\begin{array}{c}\text { ANATOMÍA } \\
\text { PATOLÓGICA }\end{array}$} & Invasión & Estroma \\
\hline & Recuento Mitótico & Moderado \\
\hline & Infiltración & Inflamatoria (+). Leucocitos mononucleares \\
\hline & Necrosis & + \\
\hline & Tamaño nuclear & Libre \\
\hline & Núcleos & Redondos \\
\hline & Nucléolos & Evidentes, escaso citoplasma \\
\hline & Vascularización & Congestivos \\
\hline \multirow{4}{*}{ INMUNOHISTOQUÍMICA } & CK7 & + \\
\hline & CK20 & - \\
\hline & EMA & + \\
\hline & Cd20 & \\
\hline $\begin{array}{l}\text { DIAGNÓSTICO POR } \\
\text { IMÁGENES }\end{array}$ & & $\begin{array}{l}\text { ECO: formación sólida, de bordes poco } \\
\text { definidos, lobulados. } 26 \text { x } 30 \mathrm{~mm} \text {. Doppler } \\
(+) \\
\text { RNM: formación nodular hiperintensa, de } \\
\text { contornos irregulares. } 38 \text { x } 22 \mathrm{~mm}\end{array}$ \\
\hline
\end{tabular}

La Foto 1 A y B corresponden a microfotografías del carcinoma indiferenciado de ovario diagnosticado en la serie. 


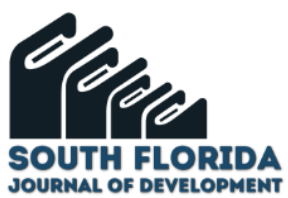

Foto 1A: Carcinoma sólido de ovario. Proliferación de células neoplásicas en cordones apretados. HE 400X. 1B: IHQ CD Evidencia las membranas citoplasmáticas positivas.400 X
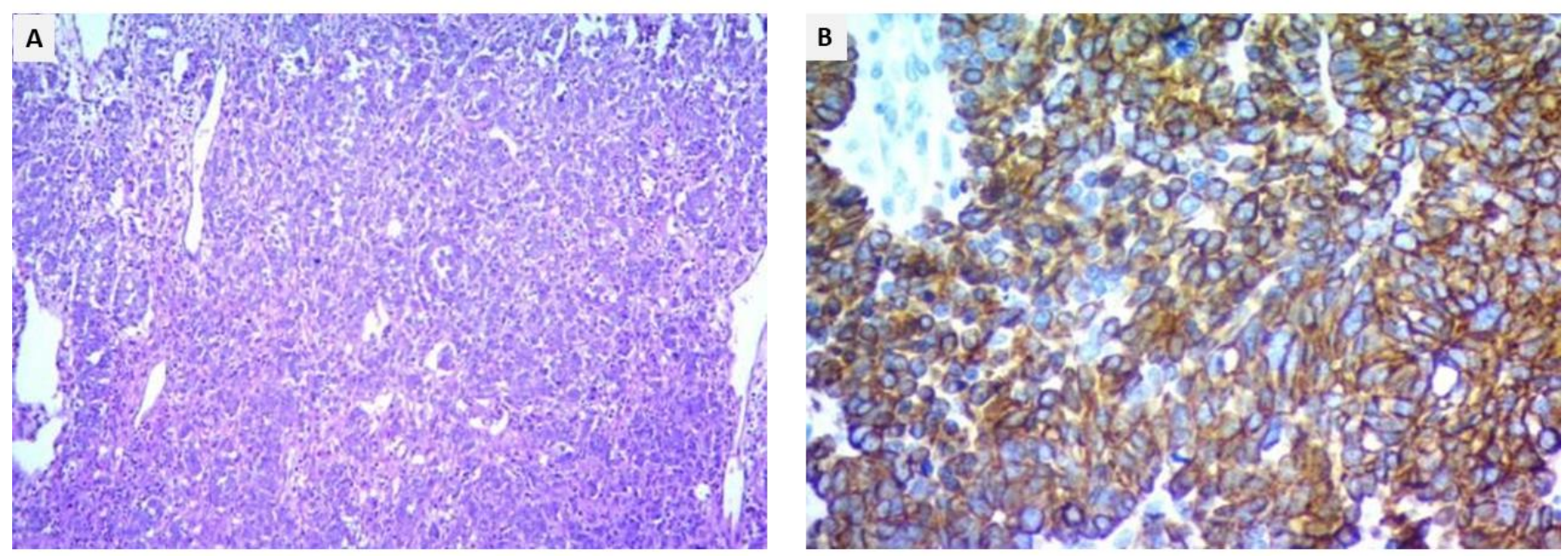

\section{DISCUSIÓN}

A partir del estudio de los tumores sólidos de ovario que fueron diagnosticados en el HNC en el período comprendido entre 2014 a 2020.

se puso de manifiesto que la ocurrencia de $\mathrm{CO}$ fue $3,8 \%$.

El CO sigue siendo la primera causa de muerte dentro de las neoplasias ginecológicas. Se diagnostica en el 65-70\% de los casos en estadios avanzados, porque su sintomatología es tardía e inespecífica $^{(12)}$

En consonancia con la bibliografía consultada, los resultados de este caso muestran la posmenospausia y la residencia en lugares industrializados como una característica. ${ }^{(13,14)}$.

En el contexto del abordaje para el diagnóstico y tratamiento del CO se incluye: identificación de la lesión, caracterización, optimización del procedimiento quirúrgico y la estrategia terapéutica ${ }^{(15)}$. La identificación de las lesiones se basa en el reconocimiento por ecografía transvaginal con cifras del 85$100 \%$ de sensibilidad y un $52-100 \%$ de especificidad para el diagnóstico de cáncer ${ }^{(16,17,18)}$. En el caso presentado y como estudios complementarios, la ecografía transvaginal fue orientadora en el diagnóstico al igual que la resonancia nuclear magnética (RNM).

Los diferentes subtipos histológicos son importantes, pues determinan el comportamiento biológico, forma de presentación, fácil diseminación, patrones de quimiorresistencia y con ello su pronóstico. Los CO indiferenciados son de peor pronóstico. ${ }^{(19,20,21)}$

A través de la determinación de biomarcadores podemos establecer la precisión diagnóstica para el control evolutivo del diagnóstico y valoración de respuesta al tratamiento. ${ }^{(22)}$ 
Las técnicas inmunohistoquímicas constituyen un recurso complementario al estudio histológico convencional. Los carcinomas de ovario presentan un patrón de expresión diferente según el tipo histológico, así los mucinosos son positivos para ambas citoqueratinas (marcadores útiles en su tipificación), mientras que los endometrioides y serosos son positivos para ck7 y negativos para ck20(23).

Entre los marcadores más útiles para la tipificación de neoplasias de ovario, se cuentan las citoqueratinas, en el caso analizado, el CK7 expresó en forma positiva. Sin embargo, la sensibilidad y especificidad no alcanza $100 \%$, debiendo ser complementadas con otros marcadores en algoritmos diagnósticos, se realizó valorando la expresión del EMA que también expresó positividad. Estos datos permitieron interpretar en conjunto con la clínica la naturaleza indiferenciada del tumor en estudio. Es esencial aclarar el diagnóstico, ya que existen tratamientos específicos.

\section{CONCLUSIONES}

Arribar al diagnóstico de CO constituye un acto médico complejo, por lo que ante la sospecha de masa pélvica y/o de un síndrome de predisposición hereditaria al cáncer, corresponde informar a la paciente sobre el riesgo de ser portadora de una mutación y la posibilidad de desarrollar la patología para ofrecer las recomendaciones adecuadas. ${ }^{(24)}$ Se debe plantear el estudio genético y es la paciente quien, debe tomar su propia decisión. ${ }^{(25,26)}$

De acuerdo a lo reportado en la bibliografía científica el $90 \%$ de los CO son de estirpe epitelial, siendo el resultado de este estudio coincidente con dicha evidencia.

La mayoría son pacientes postmenopáusicas al momento del diagnóstico. Es importante que entre los aspectos anatomopatológicos figuren un detallado estudio macroscópico, microscópico, cambios no neoplásicos y compromiso de otros órganos vecinos, así como ganglios linfáticos y realización la inmunohistoquímica. $^{(27)}$

En el diagnóstico definitivo de CO, la interpretación de los resultados debe tener en cuenta la clínica, la imagenología minuciosa, la histología y la inmunohistoquímica. 


\section{BIBLIOGRAFÍA}

1. Instituto Nacional del Cáncer Ministerio de Salud de la Nación Sala de situación del Cáncer en la República Argentina 2016 R Argentina. Acceso 10 de agosto 2020.

Disponible en: http://bancos.salud.gob.ar/sites/default/files/2018-10/0000000749cnt-63-situacion-delcanver-en-argentina-2016.pdf

2. Lowe KA, et al. (2013). An international assessment of ovarian cancer incidence and mortality. Gynecologic Oncology. 130 107-114.

3. Sharma A, et al. (2007). Symptoms in the diagnosis of ovarian cancer. In: The yearbook of obstetrics and gynaecology. T Hillard, ed. 159-70.

4. Ferlay J, et al. (2013) GLOBOCAN 2012 v1.0. Cancer incidence and mortality worldwide. IARC Cancer Base No 11.

5. American Cancer Society Tratamiento del cáncer ovárico epitelial invasivo según la etapa junio 10, 2020.

Disponible:https://www.cancer.org/es/cancer/cancer-de-ovario/tratamiento/segun-la-etapa.html

6. Maringe C, et al. (2012). Stage at diagnosis and ovarian cancer survival: evidence from the International Cancer Benchmarking Partnership. Gynecol Oncol;127:75-82.

7. Timmerman D, et al. (2010) Simple ultrasound rules to distinguish between benign and malignant adnexal masses before surgery: prospective validation by IOTA group. BMJ; 341:c6839.

8. Amor F, et al. (2011). GI-RADS reporting system for ultrasound evaluation of adnexal masses in clinical practice: a prospective multicenter study. Ultrasound Obstet Gynecol.; 38(4):450-5.

9. Baldwin LM, et al (2012). Vignette-based study of ovarian cancer screening: do U.S. physicians report adhering to evidence-based recommendations? Ann Intern Med.; 156(3):182-94.

10. Vaughan S, et al. (2011) Rethinking ovarian cancer: recommendations for improving outcomes. Nat Rev Cancer;11:719-25.

11. Mutch DG, Prat J: (2014) FIGO staging for ovarian, fallopian tube and peritoneal cancer. Gynecol Oncol 133 (3): 401-4.

12. Jayson GC, et al. (2014). Ovarian cancer. Lancet; 384: 1376-138.

13. González-Martín, A. (2015) El cáncer ginecológico. Arbor. Sociedad Española de Oncología Médica (SEOM) Vol 191. N 773.

14. Reid BM, et al (2017) Epidemiology of ovarian cancer: a review. CancerBiolMed; 14: 9-32.

15. Hernández D y González Y. (2015) Carcinomas epiteliales del ovario de alto y bajo grado. Repert.med.cir; 24(2): 105-112. 
16. Pérez-López FR, et al. (2005) Peri- and post-menopausal incidental adnexal masses and the risk of sporadic ovarian malignancy: new insights and clinical management. https://doi.org/10.3109/09513590.2010.487611.Balachandran A, Iyer RB. Imagingofovariancancer. ApplRadiol; 34: 19-29.

17. A. González M, et al. (2013) GEICO (Spanish Group for Investigation on Ovarian Cancer) treatment guidelines in ovarian cancer 2012. New Trends in Clinical Oncology Clinical and Translational Oncology Mar 07. DOI 10.1007/s12094-012-0995-8.

18. Nowak M, et al. (2015). Current clinical application of serum biomarkers to detect ovarian cancer. PrzMenopauzalny; 14(4):254-9.

19. Prat J. FIGO Committee on Gynecologic Oncology (2015). Staging classification for cancer of the ovary, fallopian tube, and peritoneum: abridged republication of guidelines from the International Federation of Gynecology and Obstetrics (FIGO) ObstetGynecol; 126:171-174.

20. Nakonechny, et al. (2016) Classification of High-Grade Epithelial Carcinomas of the Ovary. Diagnostic Accuracy, Reproducibility, and Use of Ancillary Testing. AJSP: Reviews \& Reports. - Volume 21 - Issue 4 - p 175-180.

21. American Cancer Society. Cancer Facts and Figures (2017). Atlanta, GA: American CancerSociety

22. Dabbs D, Silverman J. (2001) Immunohistochemical workup of metastatic carcinoma of unknown primary. Pathol Case Rev 6: 146-53.

23. Walsh T, et al. (2006) Spectrum of mutations in BRCA1, BRCA2, CHEK2, and TP53 in families at high risk of breast cancer. JAMA; 295(12):1379-1388.

24. Kurian AW, et al. (2017) Breast and ovarian cancer penetrance estimates derived from germline multiple-gene sequencing results in women. JCO Precision Oncology 2017; first published online June 27.

25. U.S. Preventive Services Task Force. Risk Assessment, Genetic Counseling, and Genetic Testing for BRCA-Related Cancer in Women: Clinical Summary of USPSTF Recommendation. AHRQ Publication (2013) No. 12-05164-EF-3.

26. Mariño IP, et al. (2017) Change-point of multiple biomarkers in women with ovarian cáncer, Biomed. Signal Process. Control 33, 169-177.

27. Moore RG, et al (2011) Evaluation of the diagnostic accuracy of the risk of ovarian malignancy algorithm in women with a pelvic mass. ObstetGynecol; 118(2 Pt1):280-8. 